\title{
Effects of X-ray and extreme UV radiation on circumbinary planets
}

\author{
J. Sanz-Forcada ${ }^{1}$, S. Desidera ${ }^{2}$, and G. Micela ${ }^{3}$ \\ ${ }^{1}$ Departamento de Astrofísica, Centro de Astrobiología (CSIC-INTA), ESAC Campus, PO Box 78, 28691 Villanueva de la Cañada, \\ Madrid, Spain \\ e-mail: jsanz@cab.inta-csic.es \\ 2 INAF-Osservatorio Astronomico di Padova, Italy \\ e-mail: silvano.desidera@oapd.inaf.it \\ 3 INAF-Osservatorio Astronomico di Palermo G. S. Vaiana, Piazza del Parlamento 1, Palermo, 90134 Italy \\ e-mail: giusi@astropa.inaf.it
}

Received 11 December 2013 / Accepted 15 August 2014

\begin{abstract}
Context. Several circumbinary planets have recently been discovered. The orbit of a planet around a binary stellar system poses several dynamic constraints. In addition to these constraints, the effects that radiation from the host stars may have on the planet atmospheres must be considered. We here evaluate these effects. Because of the configuration of a close binary system, these stars have a high rotation rate, even for old stars. The fast rotation of close, tidally locked binaries causes a permanent state of high stellar activity and copious XUV radiation. The accumulated effects are stronger than for normal exoplanets around single stars and cause a faster evaporation of their atmospheres.

Aims. We evaluate the effects that stellar radiation has on the evaporation of exoplanets around binary systems and on the survival of these planets.

Methods. We considered the X-ray and EUV spectral ranges (XUV, 1-912 $\AA$ ) to account for the photons that are easily absorbed by a planet atmosphere that is mainly composed of hydrogen. A more complex atmospheric composition is expected to absorb this radiation more efficiently. We used direct X-ray observations to evaluate the energy in the X-rays range and coronal models to calculate the (nondetectable) EUV part of the spectrum.

Results. We considered in this problem different configurations of stellar masses, and a resonance of 4:1 and 3:1. The simulations show that exoplanets orbiting close binary systems in a close orbit will suffer strong photoevaporation that may cause a total loss of atmosphere in a short time. We also applied our models to the best real example, Kepler- $47 \mathrm{~b}$, to estimate the current mass-loss rates in circumbinary planets and the accumulated effects over the time.

Conclusions. A binary system of two solar-like stars will be highly efficient in evaporating the atmosphere of the planet (less than $6 \mathrm{Gyr}$ in our case). These systems will be difficult to find, even if they are dynamically stable. Still, planets may orbit around binary systems of low mass stars for wider orbits. Currently known circumbinary planets are not substantially affected by thermal photoevaporation processes, unless Kepler- $47 \mathrm{~b}$ has an inflated atmosphere. The distribution of the orbital periods of circumbinary planets is shifted to much longer periods than the average of Kepler planets, which supports a scenario of strong photoevaporation in close-in circumbinary planets.
\end{abstract}

Key words. planet-star interactions - planetary systems - binaries (including multiple): close - stars: coronae - X-rays: stars stars: individual: Kepler-47

\section{Introduction}

The recent discovery of several circumbinary planets (e.g., Doyle et al. 2011; Orosz et al. 2012b) opens an interesting debate on the formation and evolution mechanism of these planets and the possible diversity of their properties compared with planets orbiting single stars. A close binary companion (separation $\$ 100$ a.u.) around a solar-type star was shown to alter both the frequency and the characteristics of giant planets (Bonavita \& Desidera 2007; Desidera \& Barbieri 2007). The orbit of a planet around a binary stellar system poses several dynamic constraints. Several works have analyzed the long-term stability of such a system (Dvorak et al. 1989; Holman \& Wiegert 1999, and references therein). A recent study (Schwarz et al. 2011) analyzed the dynamical stability of circumbinary planets using the models described in Fig. 1. There are, in general, three types of orbits in which we can find a planet around two stars:

- S-Type: the planet orbits one of the stars in a wide binary system (e.g., GJ 86 b).

- P-Type: the planet orbits the entire binary (e.g., Kepler 35 b).

- L-Type: the planet is close to the Lagrangian points L4 or L5 (Trojan planets), as in some satellites in the solar system.

The analysis of the dynamical stability yields restrictions on different variables of the problem. We focus here on P-type systems. The binary system variables, such as semimajor axis, stellar masses, or eccentricity, impose a minimum value of the semimajor axis that allows the stability of the planet orbit in the long term (Holman \& Wiegert 1999). In particular, planets with a $3: 1$ resonance with the binary system $\left(P_{\text {orb }}=3 P_{\text {bin }}\right.$ or $\left.a_{\mathrm{p}}=2.08 a_{\mathrm{bin}}\right)$ are unlikely to be stable in the long term, 


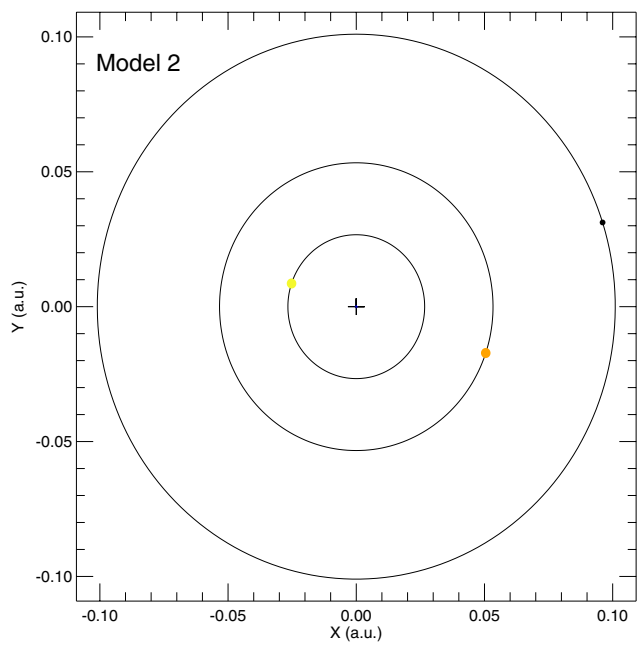

Fig. 1. Orbit of model $2\left(0.5 M_{\odot}+1.0 M_{\odot}\right)$. The planet size is not to scale $\left(M_{\mathrm{p}}=1 M_{\mathrm{J}}\right)$.

even for circular orbits. The eccentricity of the planet, combined with the stellar masses, imposes additional restrictions on the planet semimajor axis: the closest planets can be found in systems with lower eccentricity and stars with similar masses. An eccentricity higher than 0.3 hampers the survival of a close-in circumbinary planet (Schwarz et al. 2011), although such planets may exist in more distant orbits. A close central binary system is also expected to exert relevant perturbations of the surrounding circumbinary disks, with strong implications for planet formation. Planet formation is most probable far from the inner edge of a circumbinary disk (Martin et al. 2013). In the inner regions the relative velocities between planetesimals are expected to prevent accretion (Paardekooper et al. 2012; Marzari et al. 2013), although in-situ formation cannot be completely ruled out in special conditions (Meschiari 2014). On the other hand, the preliminary statistical estimates based on the Kepler data set indicate that circumbinary planets are moderately frequent $(3 \%$ considering short-period planets alone, Welsh et al. 2014).

In addition to the dynamical constraints, the effects of radiation from the host stars on the planet atmospheres need to be considered. In this work we evaluate these effects. Planet gases will absorb energetic photons and eventually cause evaporation. In the long term, this evaporation may result in the total stripping of the planet atmosphere, leaving behind only a central rocky core with a small atmosphere. The mass-loss rate of the planet mainly depends on the planet composition and the radiation received. A high-density planet will resist evaporation longer, as it will do an atmosphere with heavier species. We here consider the simplest case, an atmosphere dominated by hydrogen. Photons in the X-rays and extreme ultraviolet (EUV) spectral ranges (XUV, 1-912 A) will be easily absorbed by such a planet. A more complex atmospheric composition is expected to absorb this radiation more efficiently, which does not necessarily cause a larger mass loss. We then assume that all the absorbed energy causes atmospheric evaporation, although different degrees of efficiency may be considered (see Hubbard et al. 2007; Penz \& Micela 2008; Sanz-Forcada et al. 2011; Owen \& Jackson 2012, and references therein). Additional effects such as nonthermal losses due to stellar winds (Lanza 2013) are not considered in this work.

In the standard evolution of a single star the coronal radiation is typically highest $\left(L_{X} \sim 10^{-3} L_{\text {bol }}\right)$ at young ages (10-100 Myr), when the high rotation yields a high level of activity. Then it gradually fades as the star spins down during its evolution (e.g., Favata \& Micela 2003). In close binary systems with periods shorter than a few days, tidal interaction forces the stars to maintain a high rotation rate, even for old stars. The fast rotation of close, tidally locked binaries, causes a permanent state of high stellar activity (e.g., RS CVn stars, Walter et al. 1978), and a copious XUV radiation. Chromospheric lines are typically observed in emission in these cases, including $\mathrm{Ca}$ II $\mathrm{H} \& \mathrm{~K}$, the $\mathrm{Ca}$ infrared triplet (IRT), and $\mathrm{H} \alpha$ (e.g., Strassmeier et al. 1988; Montes et al. 2000). The stellar corona is more sensitive to activity, with copious emission in the X-rays and EUV bands in close binary systems (Pallavicini et al. 1981; Dempsey et al. 1993; Dupree et al. 1993; SanzForcada et al. 2003). The accumulated effects are stronger than for exoplanets orbiting single stars at the same distance of the parent star, and cause a faster evaporation of their atmospheres. Evidence of atmospheric evaporation has been reported in planets around single stars (Sanz-Forcada et al. 2010, and references therein). Stellar XUV radiation decays quickly over time in single stars, therefore it is expected that effects are stronger in planets around close binaries.

We carried out numerical simulations with different configurations of stellar masses to test these effects (Sect. 2), with early results presented in Sanz-Forcada et al. (2012). In Sect. 3 we apply our simulations to the currently known circumbinary systems to estimate the current mass-loss rates in circumbinary planets and the accumulated effects over time. In Sect. 4 we discuss the results and consider the minimum distance at which a circumbinary planet may survive; we conclude in Sect. 5.

\section{Numerical simulations}

The mass-loss rate due to atmospheric evaporation depends on the high-energy radiation received. Before we select a model for a mass-loss rate we need to evaluate $F_{\mathrm{XUV}}$, the X-ray and EUV flux received at the planet position. For this purpose we considered the general case of a planet orbiting a binary stellar system, without eccentricity in either orbit, and in resonant orbits (the ratio $P_{\text {orb }} / P_{\text {bin }}$ is an integer). The flux received at the planet in that case is (see Appendix A)

$F=\frac{1}{4 \pi}\left(\frac{L_{\mathrm{XUV}, 1}}{a_{\mathrm{p}}^{2}-a_{1}^{2}}+\frac{L_{\mathrm{XUV}, 2}}{a_{\mathrm{p}}^{2}-a_{2}^{2}}\right)$,

where $L_{\mathrm{XUV}, 1,2}$ is the XUV luminosity of each star, $a_{\mathrm{p}}$ is the semimajor axis of the planetary orbit, and $a_{1}$ and $a_{2}$ are related to the binary semimajor axis and the stellar masses following

$a_{1}=\frac{2 a}{1+\frac{M_{1}}{M_{2}}}, \quad a_{2}=\frac{2 a}{1+\frac{M_{2}}{M_{1}}}$.

We assumed that rotational and orbital periods of the two stars are synchronized, forcing a high rotation rate, as is usual in tidally locked stellar binaries. Tidal locking is favored by small stellar separation, and it yields a faster spin until it couples the orbital period, on a time scale that is shorter for bodies of similar masses (Zahn 1977; Zahn \& Bouchet 1989; Schrijver \& Zwaan 2000, and references therein). The synchronization time has a dependence of the type $\tau_{\text {sync }} \sim\left(\frac{M_{1}}{M_{2}}\right)^{2}\left(\frac{a}{R}\right)^{6}$. Most mainsequence spectroscopic binaries tend to be synchronized for periods shorter than eight days. The main effect of a faster rotation is a strong dynamo that causes a high activity level. If the rotation rate continues to be high, the activity level will remain stable. We here assumed that the rotation is fast enough to keep the 

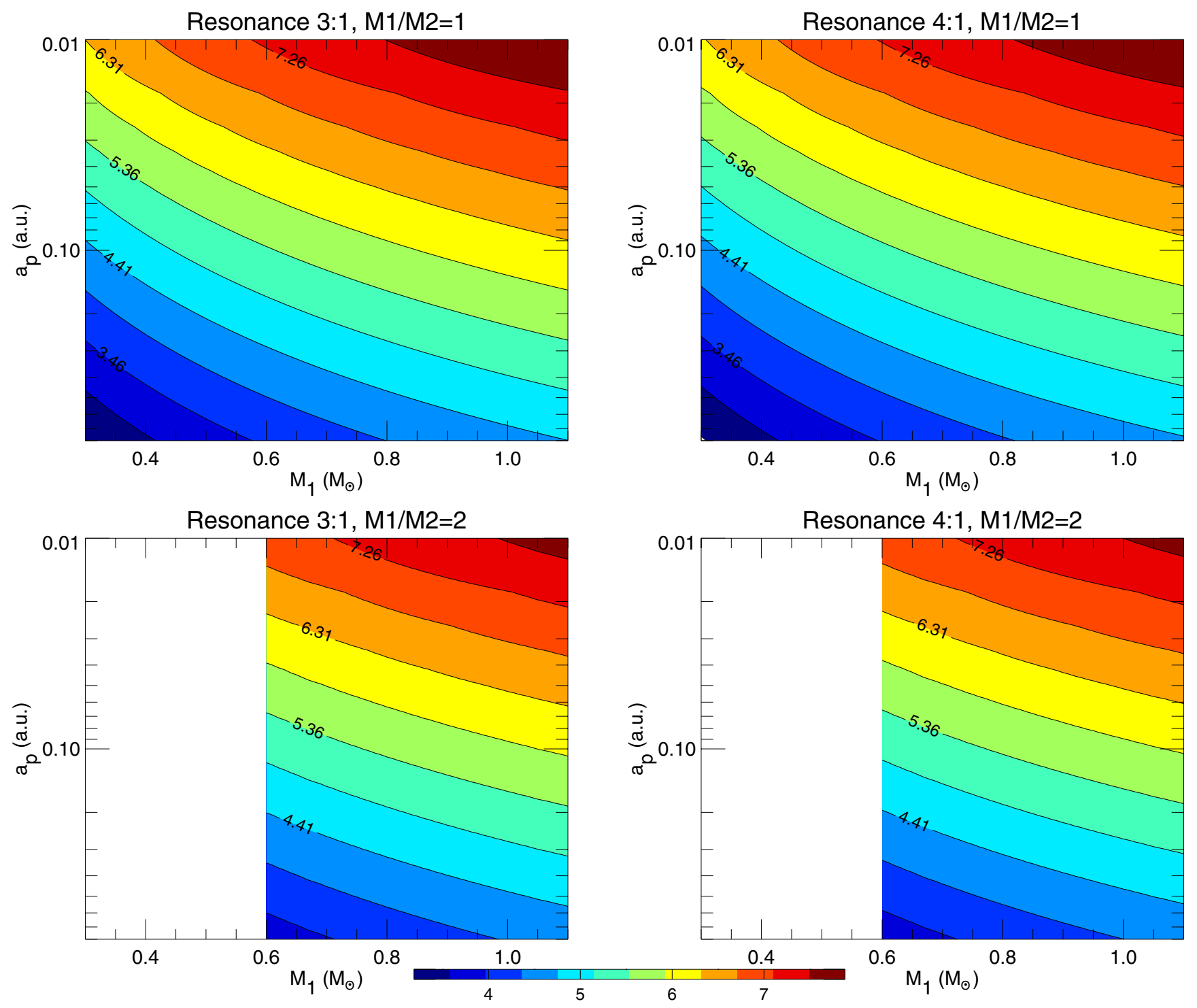

Fig. 2. Stellar XUV flux ( $\log F_{\text {XUV }}$, in $\mathrm{erg} \mathrm{s}^{-1} \mathrm{~cm}^{-2}$ ) received at the planet orbiting a binary system under activity saturation regime (color coded). Resonance 3:1 and 4:1 are considered. Upper panel: case of $M_{1}=M_{2}$. Lower panel: $M_{1}=2 M_{2}$. The planet HD 189733 b, orbiting a single star, receives $\log F_{\mathrm{XUV}}=4.2 \mathrm{erg} \mathrm{s}^{-1} \mathrm{~cm}^{-2}$ (Sanz-Forcada et al. 2011).

$\mathrm{X}$-ray emission at the saturation regime, when the $\mathrm{X}$-ray flux is highest ${ }^{1}$. The XUV luminosity of a star in the saturation regime can be related to stellar mass $\left(0.2 \lesssim M \lesssim 1.1 M_{\odot}\right)$ with this approximation (see Appendix A):

$\frac{L_{\mathrm{XUV}}}{L_{\odot}}=7.57 \times 10^{-4}\left(\frac{M}{M_{\odot}}\right)^{3.8}+130.69\left(\frac{M}{M_{\odot}}\right)^{3.268}$

Figure 2 displays the flux received by a planet orbiting a binary system with mass ratios $M_{1} / M_{2} 1$ and 2 , and resonance $3: 1$ and 4:1 (see below). As a reference, the exoplanet HD 189733 and Earth receive a $\log F_{\text {XUV }}\left(\mathrm{erg} \mathrm{s}^{-1} \mathrm{~cm}^{-2}\right) \sim 4.2$ and 0.5 respectively (Sanz-Forcada et al. 2011). In currently known circumbinary systems (e.g., Kepler-47 AB, with $P_{\text {orb }}=7.45$ d, Table 1) the activity is most likely lower than this (see Pizzolato et al. 2003), therefore assuming a saturation regime places an upper limit on the photoerosion of the planet.

\footnotetext{
1 Saturation in the X-ray flux takes place for rotation periods shorter than about 2-3 d for $\mathrm{G}$ stars, $3.3 \mathrm{~d}$ for $\mathrm{K}$ stars, and more than $10 \mathrm{~d}$ for M stars (Pizzolato et al. 2003).
}

We simulated the radiation effects for different configurations of the stellar binary system. We studied the evaporation of a Jupiter-like planet with a density of $\rho=1 \mathrm{~g} \mathrm{~cm}^{-3}\left(\rho_{\mathrm{J}}=\right.$ $1.24 \mathrm{~g} \mathrm{~cm}^{-3}$ ). Erosion and time evolution of the XUV luminosity from Sanz-Forcada et al. (2011, and references therein) are used in the calculations. The mass-loss rate due to thermal photoevaporation in a hydrogen planet atmosphere is calculated as

$\dot{M}=\frac{3 F_{\mathrm{XUV}}}{4 \mathrm{G} \rho}$

where $\mathrm{G}$ is the gravity constant, and $\rho$ is the planet density $^{2}$. This formula represents a quite simple approach to the thermal losses and probably does not truly represent the actual evaporation rate (detailed models predict an efficiency of $\lesssim 10 \%$ ). In addition, radiation levels exceeding $10^{5} \mathrm{erg} \mathrm{s}^{-1} \mathrm{~cm}^{-2}$ might slow down the increase rhythm of the evaporation rates (Murray-Clay et al. 2009). But it serves the purpose of a qualitative modeling of the problem. Finally, nonthermal losses due

2 An evolution with constant density was assumed. This assumption
does not substantially affect the conclusions (Sanz-Forcada et al. 2011). 
Table 1. Planets orbiting a binary system.

\begin{tabular}{lccccccccccc}
\hline \hline Planet name & $\begin{array}{c}M_{\mathrm{A} / \mathrm{B}} \\
M_{\odot}\end{array}$ & $\begin{array}{c}a_{\text {bin }} \\
\text { a.u. }\end{array}$ & $\begin{array}{c}a_{\mathrm{p}} \\
\text { a.u. }\end{array}$ & $\begin{array}{c}M_{\mathrm{p}}{ }^{a} \\
M_{\mathrm{J}}\end{array}$ & $e_{\mathrm{p}}$ & $P_{\text {orb }}$ & $\begin{array}{c}P_{\text {bin }} \\
\mathrm{d}\end{array}$ & $\begin{array}{c}e_{\text {bin }} \\
\mathrm{d}\end{array}$ & $\begin{array}{c}a_{\text {crit }} \\
\text { a.u. }\end{array}$ & $a_{\mathrm{p}} / a_{\text {crit }}$ & $d_{\text {periastron }} / a_{\text {crit }}$ \\
\hline Kepler 16 b & $0.69 / 0.20$ & 0.224 & 0.7048 & 0.333 & 0.007 & 228.776 & 41.08 & 0.15944 & 0.646 & 1.09 & 1.08 \\
Kepler 34 b & $1.05 / 1.02$ & 0.229 & 1.0896 & 0.220 & 0.182 & 288.822 & 27.80 & 0.52087 & 0.836 & 1.30 & 1.06 \\
Kepler 35 b & $0.89 / 0.81$ & 0.176 & 0.6035 & 0.127 & 0.042 & 131.458 & 20.73 & 0.1421 & 0.497 & 1.21 & 1.16 \\
Kepler 38 b & $0.95 / 0.25$ & 0.1469 & 0.4644 & $(0.076)$ & $<0.032$ & 105.595 & 18.80 & 0.1032 & 0.389 & 1.19 & N/A \\
Kepler 47 b & $1.04 / 0.36$ & 0.0836 & 0.2956 & $(0.024)$ & $<0.035$ & 49.514 & 7.45 & 0.0234 & 0.202 & 1.46 & N/A \\
Kepler 47 c & & & 0.989 & $(0.090)$ & $<0.411$ & 303.148 & & & & 4.90 & N/A \\
PH1 b & $1.53 / 0.41$ & 0.1744 & 0.634 & 0.53 & 0.054 & 138.506 & 20.00 & 0.2165 & 0.533 & 1.19 & 1.13 \\
Kepler 413 b & $0.82 / 0.54$ & 0.1015 & 0.355 & 0.211 & 0.118 & 66.262 & 10.12 & 0.037 & 0.260 & 1.37 & 1.20 \\
\hline
\end{tabular}

Notes. ${ }^{(a)}$ Masses in parenthesis were calculated using the planet radius and the density of Neptune $\left(1.61 \mathrm{~g} \mathrm{~cm}^{-3}\right)$.

References. Doyle et al. (2011); Welsh et al. (2012); Orosz et al. (2012a,b); Schwamb et al. (2013); Kostov et al. (2014).

to stellar winds (Lanza 2013) can be of the same order as the thermal losses considered here. The effect of these winds might roughly double the mass-loss rate in these exoplanets. We did not include these effects here.

To simulate the mass loss of the planets for this simple approach we considered three different models depending on the stellar masses, with the same configurations as in Schwarz et al. (2011): $0.3 M_{\odot}+0.3 M_{\odot}($ model 1$), 0.5 M_{\odot}+1.0 M_{\odot}($ model 2$)$, and $1 M_{\odot}+1 M_{\odot}$ (model 3$)$. In all cases the semimajor axes were set to $a=0.04$ and $a_{\mathrm{p}}=0.101$. This configuration results in a $4: 1$ resonance, to avoid conflict with the long-term instability of the system that might be present with a resonance $3: 1$. An eccentricity of $e=0.0$ was used in the simulations, consistently with the hypothesis of tidally locked binaries. The currently known planet with the closest orbit is Kepler-42 c ( $a=0.006$, Muirhead et al. 2012), and the circumbinary planet with shortest distance is Kepler-47 (AB) b $\left(a_{\mathrm{p}}=0.2956\right.$, Table 1$)$.

We calculated the effects of the stellar XUV flux evolution for a whole planetary period ( $15.12 \mathrm{~d}$ in model $1,9.57 \mathrm{~d}$ in model 2 , and $8.29 \mathrm{~d}$ in model 3) assuming constant XUV stellar luminosity during the period. Then we convolved the effects with the age of the system, starting at an age of $20 \mathrm{Myr}$, when most circumstellar disks are dissipated, and ending at 13.7 Gyr (approximate age of the Universe). This value was reduced to $9 \mathrm{Gyr}$ when there is at least one solar-mass star in the system, corresponding to its approximate evolution lifetime in the main sequence. As an example, the range of XUV flux received at the planetary orbit in model 2 (resonance $4: 1$ ) is $\log F_{\mathrm{XUV}}\left[\mathrm{erg} \mathrm{s}^{-1} \mathrm{~cm}^{-2}\right]=5.6-5.9$, with secondary maxima at $\log F_{\mathrm{XUV}}=5.7$ because of the different flux from the two stars. Figure 3 displays the simulation results for the three models. The highest photoevaporation is seen in the model $3\left(1 M_{\odot}+1 M_{\odot}\right)$. The absolute XUV flux from the solar-like stars is much higher than that of the M stars because of their size, resulting in a fast ( $\sim 6$ Gyr) evaporation of the Jovian planet. The planet around two M stars would survive in the long term, although the mass lost is considerable and would severely affect a planet of smaller mass.

In Fig. 3 we also show the effects in model 3 for a higher eccentricity $(e=0.3$ ) and a different planet density. We include a comparison with the normal evolution of coronal emission in single late-type stars $\left(L_{\mathrm{X}} \sim t^{-1.55}, L_{\mathrm{EUV}} \sim t^{-1.24}\right.$ after the saturation period, Sanz-Forcada et al. 2011). An eccentricity of 0.3 would result in only a slightly stronger photoerosion effect. A higher planet density substantially protects it from photoerosion, as expected from Eq. (3). The same stellar configuration, but with a normal evolution of the stellar activity would result in very weak effects for a Jovian planet. But currently known Jovian exoplanets include cases with a density as low as $\sim 0.1 \mathrm{~g} \mathrm{~cm}^{-3}$; in this case, the planet would loose the whole atmosphere in less than 4 Gyr even in model 1.

\section{Application to current circumbinary planets}

We tested the scenario of planetary mass loss due to radiation on real cases. Table 1 lists the sample of currently known P-type circumbinary planets. We excluded from the list the massive planets or brown dwarfs in a wide orbit $\left(a_{\mathrm{p}}>80\right.$ a.u. $)$ that were discovered by direct imaging (Ros 458 b, SR 12 c, 2MASS0103, FW Tau b and ROXs 42B b; Burgasser et al. 2010; Kuzuhara et al. 2011; Delorme et al. 2013; Kraus et al. 2014), because the large separation from the central stars makes the effects discussed here absolutely negligible. We also excluded binary systems with cataclysmic variables or white dwarfs (PSR B162026, HU Aqr, HW Vir, NN Ser, DP Leo, UZ For, RR Cae, and NY Vir; Sigurdsson et al. 2003; Hinse et al. 2012; Beuermann et al. 2010, 2011, 2012; Potter et al. 2011; Qian et al. 2012a,b), because the common-envelope evolution of the central system would cause a different astrophysical situation. Kepler-47 b is the circumbinary planet with the closest distance to the binary system (Table 1) and receives the highest amount of XUV radiation. Kepler-35 b orbits a pair with higher emitted flux (because of larger stellar size) than Kepler-47, but this is not enough to compensate for its larger semimajor axis.

For the test we assumed that the orbit of the binary system Kepler $47 \mathrm{AB}$ is close enough to force a tidal synchronization between orbital and rotational periods, thus yielding an XUV emission close to its maximum (an emission of $\log L_{\mathrm{XUV}}=30.98$ is assumed in Kepler-47 b). This will give us an upper limit of the mass-loss rate. The results (Fig. 4) show that the impact of XUV radiation on the mass loss depends much on the (unknown) mass of the planet. Assuming the density of Neptune ${ }^{3}$ $\left(\rho_{\mathrm{N}}=1.61 \mathrm{~g} \mathrm{~cm}^{-3}\right)$, which is appropriate for the mass of Kepler- 47 b, $1.3 \%$ of the original mass is lost after 9 Gyr (average $\dot{M}=2.12 \times 10^{9} \mathrm{~g} \mathrm{~s}^{-1}$ ). The effects are stronger for a lower mass planet, although a higher density (typical of Earthlike planets) would also protect it. Therefore we added two different scenarios based on the values observed in currently known planets with similar radii. Most of them have a mass between 1.6 and $27 M_{\oplus}\left(\rho=0.33-5.5 \mathrm{~g} \mathrm{~cm}^{-3}\right)$, which discards too high values that would correspond to planets with little or no atmosphere. In the lowest density case the planet losses a remarkable $\sim 31 \%$ of its mass.

The density of Neptune-size exoplanets is very variable. 


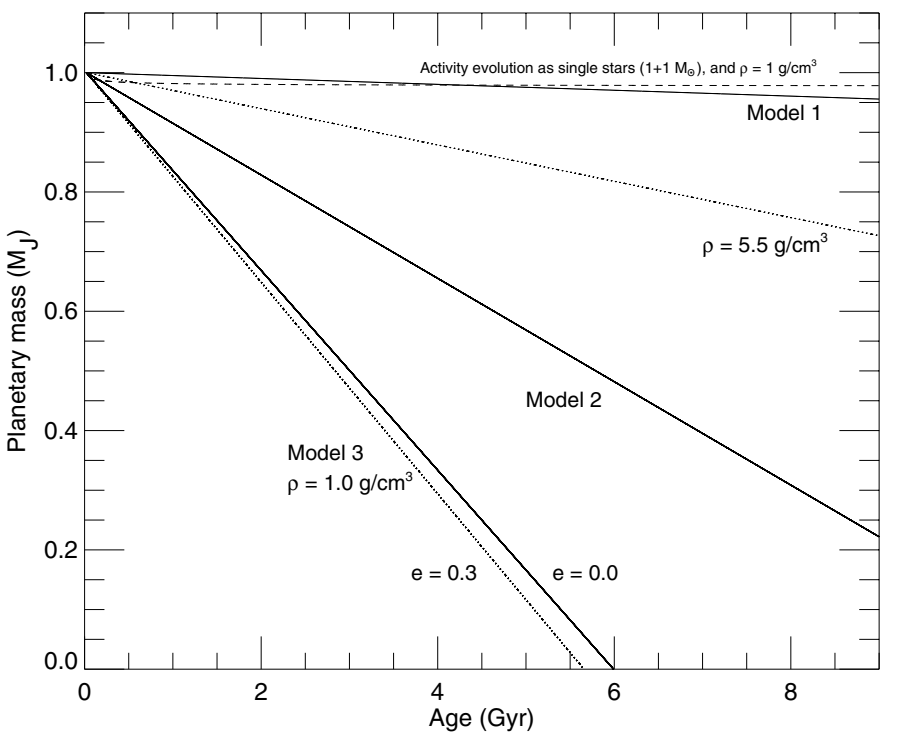

Fig. 3. Mass evolution for the different scenarios for an initial mass of $1 M_{\mathrm{J}}$, assuming a constantly high activity level. Solid lines indicate the models with an eccentricity 0.0 and a density of $1 \mathrm{~g} \mathrm{~cm}^{-3}$ (model 1: $0.3 M_{\odot}+0.3 M_{\odot}$. Model 2: $0.5 M_{\odot}+1.0 M_{\odot}$. Model 3: $\left.1 M_{\odot}+1 M_{\odot}\right)$. The effects on an Earth-like planet would be devastating even if it orbits a two M-star system. Dotted lines are used for variations of density and eccentricity in model 3. A higher density (Earth density is $5.5 \mathrm{~g} \mathrm{~cm}^{-3}$ ) protects the planet against photoevaporation. A dashed line indicates the evolution with the usual activity decay over time of single stars.

Dynamical stability in the long term due to the configuration of the binary system can be tested using the formula of Holman \& Wiegert (1999). We calculated the critical semimajor axis that allows the dynamical stability of the planet in the long term. We then compared it with the observed planet semimajor axis and its periastron for the cases in which the eccentricity has been measured. All the cases in Table 1 have both quantities quite similar to the observed semimajor axis of the planet, but still lower than it.

\section{Discussion}

Currently known circumbinary systems are not subject of substantial photoerosion by XUV radiation from central stars, unless they have a rather inflated atmosphere. To test whether we can expect to find circumbinary planets exposed to higher radiation, we considered the shortest distance at which a planet can survive the effects of radiation, depending on the stellar and planetary masses, and the density of the planet. The evaporation rates from Eq. (3) were used as a first approximation to the problem.

We considered the three models of stellar masses $\left(1+1 M_{\odot}\right.$, $0.5+1 M_{\odot}$ and $\left.0.3+0.3 M_{\odot}\right)$ and two types of planets: a Jovian planet $\left(1 M_{\mathrm{J}}\right)$ and a Neptunian planet $\left(0.054 M_{\mathrm{J}}\right.$ or $\left.17.15 M_{\oplus}\right)$. Experimental data show that Jovian-mass planets have densities below $1.3 \mathrm{~g} \mathrm{~cm}^{-3}$, and close-in planets tend to be inflated, possibly because of the XUV absorbed radiation (e.g., Sanz-Forcada et al. 2011, but other explanations have been proposed), therefore we used densities of $1.3,0.8$ and $0.3 \mathrm{~g} \mathrm{~cm}^{-3}$. For the Neptunian planet we used 2, 1 , and $0.5 \mathrm{~g} \mathrm{~cm}^{-3}$ (Neptune has $\left.\rho=1.6 \mathrm{~g} \mathrm{~cm}^{-3}\right)$. The results are displayed in Table 2 . We include the results for the scenarios with resonance $4: 1$ and 3:1.

The results show that planets with a short orbital period may suffer the effects of radiation to a great extent, which causes its

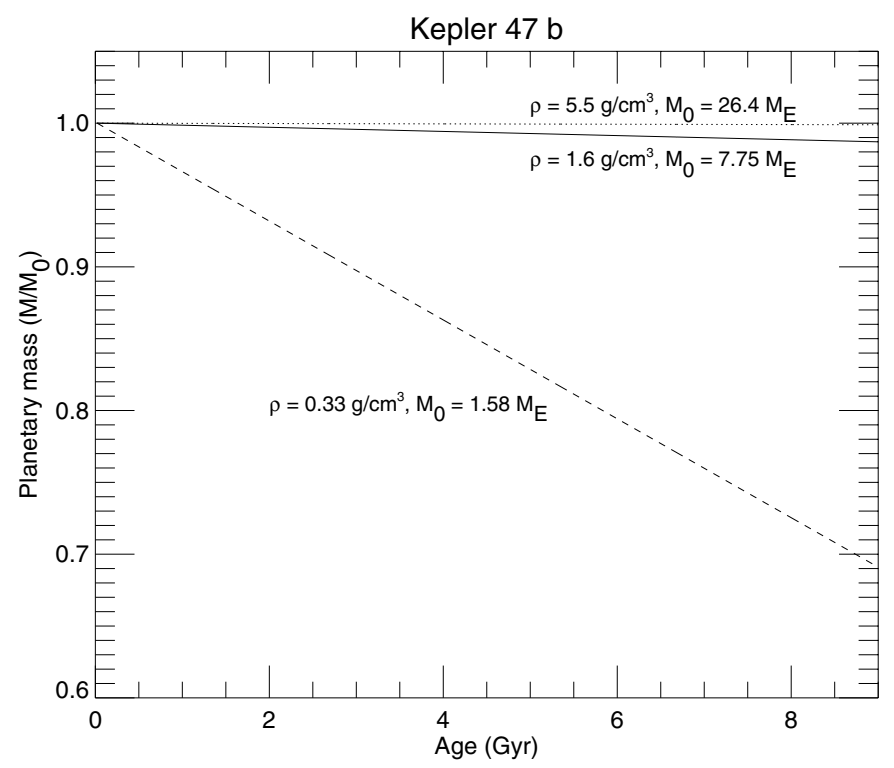

Fig. 4. Mass evolution of Kepler-47 b with age with respect to initial mass for three different densities (mass expressed in Earth units). The solid line marks a Neptune-like density. Dashed and dotted lines indicate the evolution in extreme cases of density. This is the most favorable system discovered to date for the scenario of planet photoevaporation.

total evaporation at a distance of 0.168 a.u. in 5 Gyr for an inflated Jupiter-like planet (in resonance 4:1), depending on stellar mass. Neptunian planets have even lower possibilities of survival (below $a_{\mathrm{p}}=0.562$ if $\rho=0.5 \mathrm{~g} \mathrm{~cm}^{-3}$ ). There are two limitations that need to be considered with this values: first, a higher value of $a_{\mathrm{p}}$ also implies a larger semimajor axis in the binary system ( $a=0.223$ a.u. for the mentioned Neptunian example), which means that rotation and orbital motions are not as highly coupled in the stars; the second is the long-term stability of the system, depending on the critical value of the binary star's semimajor axis, eccentricity, and masses. Equation (3) of Holman \& Wiegert (1999) reveals that P-type planets in an orbit with resonance $3: 1\left(a_{\mathrm{p}}=2.08 a_{\text {bin }}\right)$ are very unlikely to last in the long term, while those with resonance $4: 1\left(a_{\mathrm{p}}=2.52 a_{\text {bin }}\right)$ will last only if the eccentricity is close to 0. Schwarz et al. (2011) concluded that some planets with resonance 3:1 may last long enough to be detected.

From the values in Table 2 we can conclude that circumbinary planets close to the dynamical stability limits around shortperiod tidally locked binaries can exist, and they will be subject to intense evaporation effects due to photoerosion. The most massive planets might survive longer. The shortest distances required for the circumbinary planets are at least twice as long as those of the current planet with the closest orbit (Kepler-42 c, $a_{\text {bin }}=0.006$ a.u.), depending also on the spectral type of the parent stars.

The effects of photoerosion imply that circumbinary planets with a too small orbit will be difficult to find and will likely be rocky planets, as an effect of the atmosphere stripping by the XUV radiation. We can test the validity of this hypothesis by comparing the distribution of orbital periods of planets discovered by Kepler with that of the circumbinary planets in Table 1, all of which were discovered with Kepler (Fig. 5). It is remarkable to see that while most exoplanets have a short orbital period, the Kepler circumbinary planets have longer orbital periods in all cases. A difference in orbital period between circumbinary planets and planets orbiting single stars is obviously expected 
Table 2. Smallest planet semimajor axis for its survival after 5 Gyr.

\begin{tabular}{l|ccc|ccc}
\hline \hline & \multicolumn{3}{|c}{ Jovian planet } & \multicolumn{5}{c}{ Neptunian planet } \\
Stellar masses & $\rho=1.3$ & 0.8 & 0.3 & $\rho=2$ & 1 & 0.5 \\
\hline & \multicolumn{6}{c}{ Resonance $4: 1$} \\
$1 M_{\odot}+1 M_{\odot}$ & 0.081 & 0.103 & 0.168 & 0.281 & 0.397 & 0.562 \\
$0.5 M_{\odot}+1 M_{\odot}$ & 0.058 & 0.074 & 0.121 & 0.202 & 0.286 & 0.404 \\
$0.3 M_{\odot}+0.3 M_{\odot}$ & 0.014 & 0.018 & 0.029 & 0.048 & 0.068 & 0.096 \\
& \multicolumn{6}{c}{ Resonance 3:1 } \\
$1 M_{\odot}+1 M_{\odot}$ & 0.085 & 0.108 & 0.176 & 0.294 & 0.416 & 0.588 \\
$0.5 M_{\odot}+1 M_{\odot}$ & 0.060 & 0.076 & 0.125 & 0.208 & 0.294 & 0.416 \\
$0.3 M_{\odot}+0.3 M_{\odot}$ & 0.014 & 0.018 & 0.030 & 0.050 & 0.071 & 0.101 \\
\hline
\end{tabular}

Notes. Planet densities in $\mathrm{g} \mathrm{cm}^{-3}$, planet semimajor axis in a.u.

considering the stability limits that exclude the inner regions around a binary system. The currently known circumbinary planets are mostly found close (within 20\%) to the dynamical stability limits (Table 2). This is consistent with some theoretical predictions of planet migration in circumbinary disks (Pierens $\&$ Nelson 2013). Furthermore, the detection of Kepler planets around single stars is biased toward those with a short period ( $\$ 200$ d, Petigura et al. 2013). But the different distribution of circumbinary planets in Fig. 5 is difficult to explain with these effects alone, even considering the small size of the sample in Table 1. In fact, Kepler observed a large sample of short-period eclipsing binaries (Slawson et al. 2011, their Fig. 8) and about half of the detached and semi-detached binary systems have orbital periods shorter than $5 \mathrm{~d}$. On the other hand, all the eclipsing binary systems with circumbinary planets discovered so far have an orbital period longer than $7 \mathrm{~d}$. This difference was also noted in Welsh et al. (2014), who did not ascribe this to selection effects, because close planets should be easier to discover around closer binaries because of geometrical effects and more transits. On the other hand, Welsh et al. (2014) did not mention the effects of the higher levels of magnetic activity of the closer binaries on planet detectability. A more detailed study is required to asses whether selection effects play a role in the currently observed lack of circumbinary planets around the shortest-period eclipsing binaries.

If it is real, a possible explanation which we are proposing here is that circumbinary planets form or migrated preferentially close to the dynamical stability limits (Martin et al. 2013), but they are strongly affected by evaporation effects and then reduced to compact remnants, making their detection challenging. An alternative hypothesis, also mentioned in Welsh et al. (2014), is that the closest binary systems $(P<3-5 \mathrm{~d})$ do not form circumbinary planets at all, possibly because they very often have tertiary stellar companions (Tokovinin et al. 2006) that may have played a role in the tightening of the orbit of the inner binary (e.g., through Kozai interactions, see Mazeh \& Shaham 1979). This evolution is expected to be disruptive for circumbinary planets. Future observations of circumbinary planets around close binaries and a comprehensive statistical analysis on the frequency and properties of circumbinary planets at different binary orbital periods will provide more information on the occurrence of evaporation on their circumbinary planets, and by extension, to exoplanets with close-in orbits to single stars. An observational problem is measuring the planetary mass because it is difficult to determine the radial velocity in circumbinary planets.

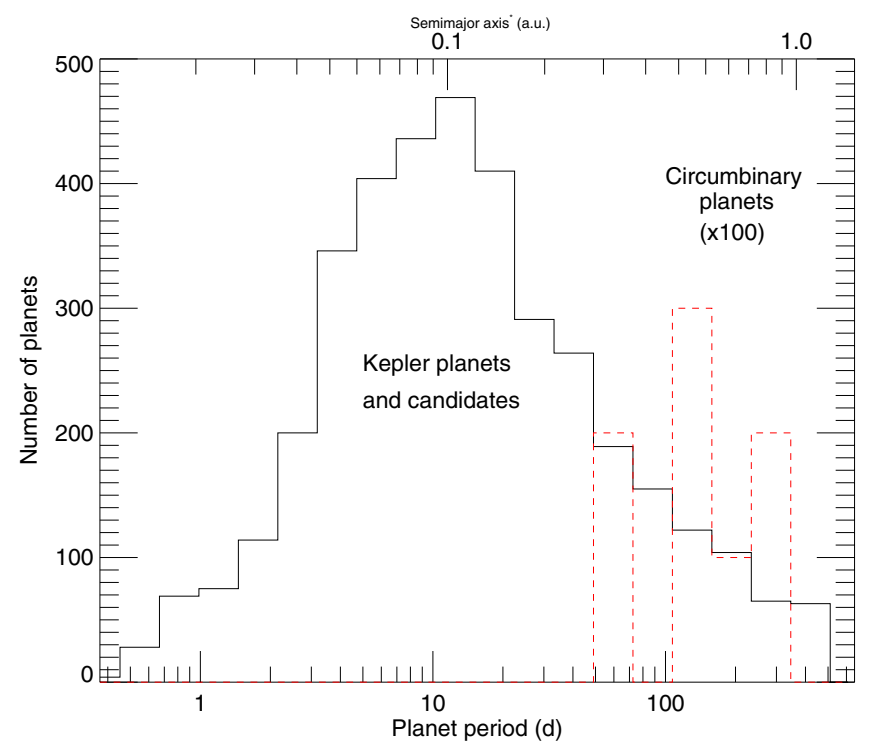

Fig. 5. Distribution of Kepler planet candidates with orbital period (solid line). The dashed line indicates the distribution of the population (multiplied $\times 100$ ) of known Kepler circumbinary planets. The upper axis marks the corresponding value of the semimajor axis calculated for a host star with $1 M_{\odot}$ and this range of periods.

\section{Conclusions}

The simulations showed that exoplanets orbiting close binary systems will suffer strong photoevaporation that may cause total loss of atmosphere in a short time, depending on the initial planet mass because of the high levels of magnetic activity that last the whole stellar lifetime.

A binary system of two solar-like stars will be highly efficient in evaporating the atmosphere of the planet. This indicates that these systems will be difficult to find, even if they are dynamically stable.

Currently known circumbinary planets are not affected by thermal photoevaporation processes, except in a scenario of an inflated atmosphere in Kepler- 47 b. The distribution of the orbital periods of Kepler circumbinary planets shows much longer periods than the average of Kepler planets, supporting the hypothesis of strong photoevaporation effects in close-in circumbinary planets.

The thermal evaporation caused by radiation we evaluated here is only part of the problem. Nonthermal processes, such as ion-picking by stellar winds, probably increase the mass-loss rate of these planets and the effects in the long term on the planetary mass.

Acknowledgements. J.S.F. acknowledges support from the Spanish MINECO through grant AYA2011-30147-C03-03. S.D. and G.M. acknowledge support from PRIN INAF 2010 "Planetary systems at young ages and the interaction with their active host stars".

\section{Appendix A: Flux received by a planet around a binary star}

To calculate the mass-loss rate due to evaporation (thermal losses) we proceeded in two steps. First we derived the stellar flux received by the planet in a given wavelength range (XUV in our case); second, we applied a model that calculates how that flux results in atmospheric evaporation, and subsequently in planet mass-loss. In this appendix we include the necessary formulae for calculating the flux received by a planet orbiting a close binary system composed of two late-type stars. 
The flux received by a planet, averaged over a whole orbital period, can be calculated from

$F=\frac{1}{4 \pi P_{\text {orb }}} \int_{t=0}^{t=P_{\text {orb }}}\left(\frac{L_{1}}{r_{1}^{2}}+\frac{L_{2}}{r_{2}^{2}}\right) \mathrm{d} t$,

where $L_{1,2}$ are the stellar luminosity in the considered wavelength range, and $r_{1,2}$ are the distances between the planet an each of the stars. Luminosities are considered constant during a period. $r_{1,2}$ are calculated by combining the movement of the stars in the system and the planet itself, resulting in

$r_{1,2}=a_{1,2}^{2}+a_{\mathrm{p}}^{2}-2 a_{1,2} a_{\mathrm{p}} \cos \left(\phi_{2}-\phi_{1}\right)$,

where $\phi_{1,2}=2 \pi \frac{t}{P_{\text {bin,orb }}}$ and $a_{\mathrm{p}}$ is the semimajor axis of the planet's orbit. $a_{1}$ and $a_{2}$ are related to the binary semimajor axis and the stellar masses following

$a_{1}=\frac{2 a}{1+\frac{M_{1}}{M_{2}}}, \quad a_{2}=\frac{2 a}{1+\frac{M_{2}}{M_{1}}}$.

We can solve the integrals by using $x=\frac{2 \pi\left(\frac{P_{\text {orb }}}{P_{\text {bin }}}-1\right) t}{P_{\text {orb }}}$ and assuming a resonant orbit $\left(P_{\text {orb }} / P_{\text {bin }}\right.$ is an integer $)$ :

$\int_{t=0}^{t=P_{\text {orb }}} \frac{\mathrm{d} t}{r_{1}^{2}}=\frac{P_{\text {orb }}}{2 \pi a_{\mathrm{p}}^{2}} \frac{2 \pi}{1-\left(a_{1} / a_{\mathrm{p}}\right)^{2}}$.

The resulting flux received at the planet's position is then

$F=\frac{1}{4 \pi}\left(\frac{L_{1}}{a_{\mathrm{p}}^{2}-a_{1}^{2}}+\frac{L_{2}}{a_{\mathrm{p}}^{2}-a_{2}^{2}}\right)$,

where $L_{1,2}$ is the luminosity of each star in a given spectral range.

We are interested in the XUV spectral range. The luminosity of a late-type star under the saturation regime can be approximated by (Sanz-Forcada et al. 2011):

$L_{\mathrm{XUV}}=6.3 \times 10^{-4} L_{\mathrm{bol}}+111.54 L_{\mathrm{bol}}^{0.86}$,

which combined with the classical luminosity-mass relation (Cox 2000)

$\log \frac{L_{\mathrm{bol}}}{L_{\odot}}=3.9 \log \left(\frac{M}{M_{\odot}}\right)+0.08$

yields

$\frac{L_{\mathrm{XUV}}}{L_{\odot}}=7.57 \times 10^{-4}\left(\frac{M}{M_{\odot}}\right)^{3.8}+130.69\left(\frac{M}{M_{\odot}}\right)^{3.268}$.

This equation is valid in the range $0.2 \lesssim M \lesssim 1.1 M_{\odot}$.

\section{References}

Beuermann, K., Hessman, F. V., Dreizler, S., et al. 2010, A\&A, 521, L60 Beuermann, K., Buhlmann, J., Diese, J., et al. 2011, A\&A, 526, A53
Beuermann, K., Dreizler, S., Hessman, F. V., \& Deller, J. 2012, A\&A, 543, A138 Bonavita, M., \& Desidera, S. 2007, A\&A, 468, 721

Burgasser, A. J., Simcoe, R. A., Bochanski, J. J., et al. 2010, ApJ, 725, 1405

Cox, A. N. 2000, Allen's astrophysical quantities, (Springer)

Delorme, P., Gagné, J., Girard, J. H., et al. 2013, A\&A, 553, L5

Dempsey, R. C., Linsky, J. L., Fleming, T. A., \& Schmitt, J. H. M. M. 1993 ApJS, 86, 599

Desidera, S., \& Barbieri, M. 2007, A\&A, 462, 345

Doyle, L. R., Carter, J. A., Fabrycky, D. C., et al. 2011, Science, 333, 1602

Dupree, A. K., Brickhouse, N. S., Doschek, G. A., Green, J. C., \& Raymond, J. C. 1993, ApJ, 418, L41

Dvorak, R., Froeschle, C., \& Froeschle, C. 1989, A\&A, 226, 335

Favata, F., \& Micela, G. 2003, Space Sci. Rev., 108, 577

Hinse, T. C., Lee, J. W., Goździewski, K., et al. 2012, MNRAS, 420, 3609

Holman, M. J., \& Wiegert, P. A. 1999, AJ, 117, 621

Hubbard, W. B., Hattori, M. F., Burrows, A., Hubeny, I., \& Sudarsky, D. 2007, Icarus, 187,358

Kostov, V. B., McCullough, P. R., Carter, J. A., et al. 2014, ApJ, 784, 14

Kraus, A. L., Ireland, M. J., Cieza, L. A., et al. 2014, ApJ, 781, 20

Kuzuhara, M., Tamura, M., Ishii, M., et al. 2011, AJ, 141, 119

Lanza, A. F. 2013, A\&A, 557, A31

Martin, R. G., Armitage, P. J., \& Alexander, R. D. 2013, ApJ, 773, 74

Marzari, F., Thebault, P., Scholl, H., Picogna, G., \& Baruteau, C. 2013, A\&A 553, A71

Mazeh, T., \& Shaham, J. 1979, A\&A, 77, 145

Meschiari, S. 2014, ApJ, 790, 41

Montes, D., Fernández-Figueroa, M. J., De Castro, E., et al. 2000, A\&AS, 146, 103

Muirhead, P. S., Johnson, J. A., Apps, K., et al. 2012, ApJ, 747, 144

Murray-Clay, R. A., Chiang, E. I., \& Murray, N. 2009, ApJ, 693, 23

Orosz, J. A., Welsh, W. F., Carter, J. A., et al. 2012a, ApJ, 758, 87

Orosz, J. A., Welsh, W. F., Carter, J. A., et al. 2012b, Science, 337, 1511

Owen, J. E., \& Jackson, A. P. 2012, MNRAS, 425, 2931

Paardekooper, S.-J., Leinhardt, Z. M., Thébault, P., \& Baruteau, C. 2012, ApJ, 754, L16

Pallavicini, R., Golub, L., Rosner, R., et al. 1981, ApJ, 248, 279

Penz, T., \& Micela, G. 2008, A\&A, 479, 579

Petigura, E. A., Howard, A. W., \& Marcy, G. W. 2013, Proc. Nat. Acad. Sci., 110,19273

Pierens, A., \& Nelson, R. P. 2013, A\&A, 556, A134

Pizzolato, N., Maggio, A., Micela, G., Sciortino, S., \& Ventura, P. 2003, A\&A, 397,147

Potter, S. B., Romero-Colmenero, E., Ramsay, G., et al. 2011, MNRAS, 416, 2202

Qian, S.-B., Liu, L., Zhu, L.-Y., et al. 2012a, MNRAS, 422, L24

Qian, S.-B., Zhu, L.-Y., Dai, Z.-B., et al. 2012b, ApJ, 745, L23

Sanz-Forcada, J., Brickhouse, N. S., \& Dupree, A. K. 2003, ApJS, 145, 147

Sanz-Forcada, J., Ribas, I., Micela, G., et al. 2010, A\&A, 511, L8

Sanz-Forcada, J., Micela, G., Ribas, I., et al. 2011, A\&A, 532, A6

Sanz-Forcada, J., Desidera, S., \& Micela, G. 2012, in 17th Cambridge Workshop on Cool Stars, Stellar Systems and the Sun, http://www. coolstars17. net/ficha_participant.php?n=1331816056

Schrijver, C. J., \& Zwaan, C. 2000, Solar and Stellar Magnetic Activity (Cambridge University Press)

Schwamb, M. E., Orosz, J. A., Carter, J. A., et al. 2013, ApJ, 768, 127

Schwarz, R., Haghighipour, N., Eggl, S., Pilat-Lohinger, E., \& Funk, B. 2011, MNRAS, 414, 2763

Sigurdsson, S., Richer, H. B., Hansen, B. M., Stairs, I. H., \& Thorsett, S. E. 2003, Science, 301, 193

Slawson, R. W., Prša, A., Welsh, W. F., et al. 2011, AJ, 142, 160

Strassmeier, K. G., Hall, D. S., Zeilik, M., et al. 1988, A\&AS, 72, 291

Tokovinin, A., Thomas, S., Sterzik, M., \& Udry, S. 2006, A\&A, 450, 681

Walter, F., Charles, P., \& Bowyer, S. 1978, AJ, 83, 1539

Welsh, W. F., Orosz, J. A., Carter, J. A., et al. 2012, Nature, 481, 475

Welsh, W. F., Orosz, J. A., Carter, J. A., \& Fabrycky, D. C. 2014, in IAU Symp., 293, ed. N. Haghighipour, 125

Zahn, J.-P. 1977, A\&A, 57, 383

Zahn, J.-P., \& Bouchet, L. 1989, A\&A, 223, 112 